\title{
LOCALIZING WITH RESPECT TO SELF-MAPS OF THE CIRCLE
}

\author{
CARLES CASACUBERTA AND GEORG PESCHKE
}

\begin{abstract}
We describe a general procedure to construct idempotent functors on the pointed homotopy category of connected CW-complexes, some of which extend $P$-localization of nilpotent spaces, at a set of primes $P$. We focus our attention on one such functor, whose local objects are CW-complexes $X$ for which the $p$ th power map on the loop space $\Omega X$ is a self-homotopy equivalence if $p \notin P$. We study its algebraic properties, its behaviour on certain spaces, and its relation with other functors such as Bousfield's homology localization, Bousfield-Kan completion, and Quillen's plus-construction.
\end{abstract}

\section{INTRODUCTION}

Localization methods at a set of primes $P$ have been broadly used in group theory and homotopy theory during the last two decades. These methods have been applied primarily in the category of nilpotent groups and in the pointed homotopy category of nilpotent CW-complexes; see $[1,7,16]$.

If one seeks to extend $P$-localization to arbitrary $C W$-complexes, then there is in fact more than one choice available. Among the various choices, the ones which have been described in the literature are contained in the work of Bousfield [4, 5] and Bousfield-Kan [7].

In this paper, we study extensions of the $P$-localization of nilpotent $\mathrm{CW}$ complexes to all $\mathrm{CW}$-complexes from two points of view:

(1) We consider the family of all possible such extensions and provide a uniform construction of some of them, namely of those which are spliceable, in the sense explained below.

(2) Among such spliceable functors, there is one which is most intimately tied to $P$-localization of groups in the sense of $[16,22]$. We explain its basic properties.

To explain (1), we observe that the functors in our discussion are interrelated by natural transformations. In this way they form a partially ordered system containing a unique maximal and a unique minimal element. The minimal element of this system turns out to be Bousfield's $H_{*}\left(; \mathbb{Z}_{P}\right)$-localization [4], meaning that $H_{*}\left(; \mathbb{Z}_{P}\right)$-localization factors uniquely through every extension of $P$-localization of nilpotent spaces to all spaces.

The $H_{*}\left(; \mathbb{Z}_{P}\right)$-localization functor is characterized by the class of maps which become homotopy equivalences after localization, namely all maps which

Received by the editors May 25, 1991.

1980 Mathematics Subject Classification (1985 Revision). Primary 55P60; Secondary 55N25, 55P10, 55P35. 
induce an isomorphism in singular homology with $\mathbb{Z}_{P}$ coefficients. The functors which we describe render invertible all maps inducing at the fundamental group a homomorphism from a specially selected class and an isomorphism in homology with certain twisted coefficients. The construction of these functors involves two processes. Firstly, homology localization in the sense of Bousfield in categories of the following type: an object consists of a space together with a twisted coefficient system, and a morphism is a map compatible with the coefficient systems; see $\S 5$. Secondly, "splicing" such functors in order to obtain idempotent functors on the pointed homotopy category of CW-complexes; see $\S 6$.

While splicing affords a uniform point of view of $P$-localization through the eyes of homology, we emphasize that some of our functors have independent descriptions and constructions which are more direct and geometrically inspired. One nontrivial result seen with the aid of such a double description of a localizing functor is that homology and cohomology equivalences with certain $P$-local twisted coefficients coincide (Corollary 7.3).

We now turn to the special $P$-localizing functor $X \rightarrow X_{P}$ mentioned in (2) above. Recall that a group $G$ is called $P$-local if for all primes $n \notin P$ the power function $x \mapsto x^{n}$ is a bijection-or, equivalently, a homotopy equivalence, regarding $G$ as a discrete space. The distinguishing feature of our special localizing functor is that its local spaces have loop spaces on which the power function $\omega \mapsto \omega^{n}, n \notin P$, is a homotopy equivalence; i.e. $P$-local spaces are classifying spaces of $\left(A_{\infty}\right.$-associative $) P$-local $H$-groups. They are also characterized by the property that $\pi_{1}(X)$ is a $P$-local group acting $P$-locally on the higher homotopy groups $\pi_{i}(X)$, i.e. each element $1+x+\cdots+x^{n-1} \in \mathbb{Z}\left[\pi_{1}(X)\right]$, $n \notin P$, acts as an isomorphism on $\pi_{i}(X), i \geq 2$; cf. [19].

To deal with this kind of actions, we use the localization $P[G]$ of the integral group ring $\mathbb{Z} G$ of a $P$-local group $G$ with respect to the elements $1+x+\cdots+$ $x^{n-1}, x \in G, \quad n \notin P$, in the ring-theoretical sense [13]. Abelian groups on which $G$ acts $P$-locally are then identified as $P[G]$-modules. This is done in $\S 2$, where we also establish some foundational facts concerning $P[G]$-modules.

In $\S 3$, we provide an explicit construction of the functor $X \rightarrow X_{P}$ in countably many steps, and proceed to collect its basic properties. In $\S 4$, we prove that this functor extends $P$-localization of nilpotent spaces. Finally, in $\S 8$, we discuss its interrelation with other extensions of $P$-localization of nilpotent spaces and determine its effect on special spaces and maps, in particular on a wedge of circles.

\section{CATEgorical PRELIMinaries}

In order to explain the interrelation between various localizing functors known from the literature and new ones obtained in this paper, we invoke the general facts concerning localizing functors and orthogonal pairs described in $[11,12]$. For the convenience of the reader, we collect here what is needed. Let $\mathscr{C}$ denote a given category.

Definition 1.1. An object $X$ and a morphism $f: A \rightarrow B$ in $\mathscr{C}$ are said to be orthogonal if the function $f^{*}: \operatorname{Mor}(B, X) \rightarrow \operatorname{Mor}(A, X)$ is bijective.

For a class of morphisms $\mathscr{M}, \mathscr{M}^{\perp}$ denotes the class of objects which are orthogonal to each $f \in \mathscr{M}$. Similarly, for a class of objects $\mathscr{O}, \mathscr{O}^{\perp}$ denotes 
the class of morphisms which are orthogonal to each object $X \in \mathscr{O}$.

Definition 1.2. An orthogonal pair $(\mathscr{S}, \mathscr{D})$ in $\mathscr{C}$ consists of a class of morphisms $\mathscr{S}$ and a class of objects $\mathscr{D}$ such that $\mathscr{D}^{\perp}=\mathscr{S}$ and $\mathscr{S}^{\perp}=\mathscr{D}$.

The operation $\perp$ satisfies $\perp \perp \perp=\perp$, implying that $\left(\mathscr{M}^{\perp \perp}, \mathscr{M}^{\perp}\right)$ and $\left(\mathscr{O}^{\perp}, \mathscr{O}^{\perp \perp}\right)$ are orthogonal pairs for every class of morphisms $\mathscr{M}$, resp. of objects $\mathscr{O}$, in $\mathscr{C}$.

Localizations of $\mathscr{C}$ are described by means of idempotent monads [1], and every idempotent monad $(E, \eta)$ determines an orthogonal pair $(\mathscr{S}, \mathscr{D})$ by

$$
\mathscr{S}=\{f: A \rightarrow B \mid E f: E A \cong E B\}, \quad \mathscr{D}=\left\{X \mid \eta_{X}: X \cong E X\right\} .
$$

The construction in [12] "recovers" for many orthogonal pairs $(\mathscr{S}, \mathscr{D})$ its associated idempotent monad $(E, \eta)$, provided it exists. In this situation, we refer to morphisms in $\mathscr{S}$ as E-equivalences and to objects in $\mathscr{D}$ as E-local objects.

Let $E^{\prime}$ be a localizing functor on $\mathscr{C}^{\prime}$, a subcategory of $\mathscr{C}$, and let $\left(\mathscr{S}^{\prime}, \mathscr{D}^{\prime}\right)$ denote the orthogonal pair associated with $E^{\prime}$. Possible extensions of $E^{\prime}$ over $\mathscr{C}$ are identified by orthogonal pairs $(\mathscr{S}, \mathscr{D})$ of $\mathscr{C}$ extending $\left(\mathscr{S}^{\prime}, \mathscr{D}^{\prime}\right)$, i.e. such that $\mathscr{S}^{\prime} \subseteq \mathscr{S}$ and $\mathscr{D}^{\prime} \subseteq \mathscr{D}$. Orthogonal pairs extending $\left(\mathscr{S}^{\prime}, \mathscr{D}^{\prime}\right)$ are partially ordered by the following relation: $\left(\mathscr{S}_{1}, \mathscr{D}_{1}\right) \geq\left(\mathscr{S}_{2}, \mathscr{D}_{2}\right)$ if $\mathscr{D}_{1} \supseteq \mathscr{D}_{2}$ (equivalently, if $\mathscr{S}_{1} \subseteq \mathscr{S}_{2}$ ).

Remark 1.3. Suppose that $E_{1}, E_{2}$ are localizing functors whose associated orthogonal pairs satisfy $\left(\mathscr{S}_{1}, \mathscr{D}_{1}\right) \geq\left(\mathscr{S}_{2}, \mathscr{D}_{2}\right)$. Then $E_{2}: \mathscr{D}_{1} \rightarrow \mathscr{D}_{2}$ is left adjoint to the inclusion $\mathscr{D}_{2} \rightarrow \mathscr{D}_{1}$. In particular, there is a natural transformation $E_{1} \rightarrow E_{2}$ which commutes with the localizing transformations $\eta_{1}:$ Id $\rightarrow E_{1}$, $\eta_{2}:$ Id $\rightarrow E_{2}$.

Remark 1.4. If $(\mathscr{S}, \mathscr{D})$ extends $\left(\mathscr{S}^{\prime}, \mathscr{D}^{\prime}\right)$ over $\mathscr{C}$, then

$$
\left(\left(\mathscr{S}^{\prime}\right)^{\perp \perp},\left(\mathscr{S}^{\prime}\right)^{\perp}\right) \geq(\mathscr{S}, \mathscr{D}) \geq\left(\left(\mathscr{D}^{\prime}\right)^{\perp},\left(\mathscr{D}^{\prime}\right)^{\perp \perp}\right) .
$$

Accordingly, we call $\left(\left(\mathscr{S}^{\prime}\right)^{\perp \perp},\left(\mathscr{S}^{\prime}\right)^{\perp}\right)$ the maximal extension of $\left(\mathscr{S}^{\prime}, \mathscr{D}^{\prime}\right)$ over $\mathscr{C}$ and $\left(\left(\mathscr{D}^{\prime}\right)^{\perp},\left(\mathscr{D}^{\prime}\right)^{\perp \perp}\right)$ the minimal extension.

\section{P-LOCAL GROUPS, MODULES AND SPACES}

This section contains the algebraic foundations needed to describe a certain idempotent monad in the pointed homotopy category of connected CWcomplexes. The key concept is that of a group $G$ acting $P$-locally on an abelian group $A$ (Definition 2.4). The motivation to consider such actions comes from Theorem 2.1 below.

When dealing with spaces and maps, we work in the based setting throughout. Thus $[X, Y]$ denotes the set of pointed homotopy classes of maps from $X$ to $Y$. By $P$ we always denote a (fixed) set of primes, and by $P^{\prime}$ its complement in the set of all primes. We write $n \in P^{\prime}$ if $n$ belongs to the multiplicative closure of the primes in $P^{\prime}$. A group $G$ will be called $P$-local $[16,22,27]$ if the $n$th power map $x \mapsto x^{n}$ is bijective on $G$ for every $n \in P^{\prime}$.

Theorem 2.1. For a connected $C W$-complex $X$, the following assertions are equivalent:

(a) On the loop space $\Omega X$ the nth power map $\omega \mapsto \omega^{n}$ is a self-homotopy equivalence for every $n \in P^{\prime}$. 
(b) The group $[W, \Omega X]$ is P-local for every space $W$.

(c) The groups $\pi_{1}(X)$ and $\pi_{k}(X) \rtimes \pi_{1}(X), k \geq 2$, are P-local, where $\rtimes$ denotes the semidirect product with respect to the standard action.

Proof. This is a consequence of the natural group isomorphism

$$
\pi_{k}(X) \rtimes \pi_{1}(X) \cong\left[S^{k-1} \cup \mathrm{pt}, \Omega X\right],
$$

where pt denotes a disjoint base point; see $[18,19]$.

Definition 2.2. We call P-local those connected CW-complexes $X$ satisfying the equivalent conditions of Theorem 2.1 .

Let $\operatorname{map}_{*}(A, X)$ denote the space of pointed maps from $A$ to $X$. Using condition (2.1.b) and the exponential law [8], one infers

Proposition 2.3. If $X$ is $P$-local, then the base point component of $\operatorname{map}_{*}(A, X)$ is also P-local, for every finite $C W$-complex $A$.

Note that, if a CW-complex $X$ is nilpotent, then the groups $\pi_{k}(X) \rtimes \pi_{1}(X)$ are nilpotent. Therefore, a nilpotent CW-complex $X$ is $P$-local in our sense if and only if the groups $\pi_{k}(X)$ are $P$-local for $k \geq 1$, because $P$-localization is exact in the category of nilpotent groups. Thus our terminology is consistent with the classical one [16]. Next we characterize the action of the fundamental group of a $P$-local space on the higher homotopy groups in a way which is more readily accessible to methods from Homological Algebra.

Definition 2.4. Let $G$ be a group and $A$ a (left) $\mathbb{Z} G$-module. Denote by $\omega$ : $\mathbb{Z} G \rightarrow \operatorname{End}(A)$ the associated ring homomorphism. We say that $A$ is a $P$-local $\mathbb{Z} G$-module if $\omega\left(1+x+x^{2}+\cdots+x^{n-1}\right) \in \operatorname{Aut}(A)$ for all $x \in G, n \in P^{\prime}$.

We also say that the action of $G$ on $A$ is $P$-local, or that $G$ acts P-locally on $A$. This notion is easily generalized to the case when $A$ is a group, not necessarily abelian, on which $G$ acts; see $[11,12,14,19]$. We shall often use the notation

$$
\rho_{n, x}=1+x+x^{2}+\cdots+x^{n-1} .
$$

Remark 2.5. Given a group $G$ and a $\mathbb{Z} G$-module $A$, the identity

$$
(a, x)^{n}=\left(\rho_{n, x} a, x^{n}\right), \quad n \geq 1,
$$

in the group $A \rtimes G$ shows that $A \rtimes G$ is $P$-local if and only if $G$ is a $P$-local group and $A$ is a $P$-local $\mathbb{Z} G$-module.

In the light of Remark 2.5, condition (c) in Theorem 2.1 can be reformulated as

$$
\begin{aligned}
& \pi_{1}(X) \text { is a P-local group and each } \pi_{k}(X), k \geq 2 \text {, is a P-local } \\
& \mathbb{Z}\left[\pi_{1}(X)\right] \text {-module. }
\end{aligned}
$$

Our next step is to identify $P$-local $\mathbb{Z}\left[\pi_{1}(X)\right]$-modules as modules over a certain universal ring associated to $\mathbb{Z}\left[\pi_{1}(X)\right]$, in which the elements $1+x+$ $\cdots+x^{n-1}, x \in \pi_{1}(X), \quad n \in P^{\prime}$, are units. We need to recall some general facts of ring theory. Let $R$ be a ring with 1 , and $S \subseteq R$ be an arbitrary subset. As explained in [13], there exists a ring homomorphism $\alpha: R \rightarrow R_{S}$, unique up to isomorphism, such that

(i) $\alpha(s)$ is invertible in $R_{S}$ for all $s \in S$, and 
(ii) if $f: R \rightarrow T$ is any ring homomorphism for which $f(s)$ is invertible in $T$ for all $s \in S$, then there is a unique ring homomorphism $f^{\prime}$ : $R_{S} \rightarrow T$ with $f^{\prime} \alpha=f$.

In particular, (ii) shows that $\alpha$ is a ring epimorphism, meaning that for every diagram of rings

$$
R \stackrel{\alpha}{\rightarrow} R_{S} \stackrel{u}{\stackrel{v}{\rightrightarrows}} T
$$

where $u \alpha=v \alpha$, it follows that $u=v$. Therefore [26], the category of $R_{S}$-modules embeds as a full subcategory in the category of $R$-modules via $\alpha$, and this embedding has a left adjoint $\alpha^{*}$ given by

$$
\alpha^{*}(A)=R_{S} \otimes_{R} A .
$$

We apply these considerations to the case $R=\mathbb{Z} G, \quad G$ a given group, and $S$ the multiplicative closure of the elements $\rho_{n, x}, x \in G, \quad n \in P^{\prime}$, cf. (2.2). Thus we obtain a universal ring homomorphism

$$
\alpha: \mathbb{Z} G \rightarrow(\mathbb{Z} G)_{S}
$$

inverting the elements of $S$.

Therefore, a $\mathbb{Z} G$-module $A$ is $P$-local if and only if the ring homomorphism $\omega: \mathbb{Z} G \rightarrow \operatorname{End}(A)$ factors uniquely through (2.5); i.e. $P$-local $\mathbb{Z} G$-modules are precisely $(\mathbb{Z} G)_{S}$-modules.

If the group $G$ is commutative, then $(\mathbb{Z} G)_{S}=S^{-1}(\mathbb{Z} G)$ is the usual ring of fractions [2]. In particular, of course, if $G=\{1\}$, then $(\mathbb{Z} G)_{S}=\mathbb{Z}_{P}$, the ring of integers localized at $P$.

We now collect several elementary facts concerning $(\mathbb{Z} G)_{S}$-modules which will be needed in the sequel.

Lemma 2.6. Let $G$ be a group, and

$$
0 \rightarrow A^{\prime} \rightarrow A \rightarrow A^{\prime \prime} \rightarrow 0
$$

a short exact sequence of $\mathbb{Z} G$-modules. Then, if any two of $A^{\prime}, A, A^{\prime \prime}$ are $P$ local, so is the third.

Proof. For each $n \in P^{\prime}$ and $x \in G$, the operation $\rho_{n, x}$ induces the commuting diagram below.

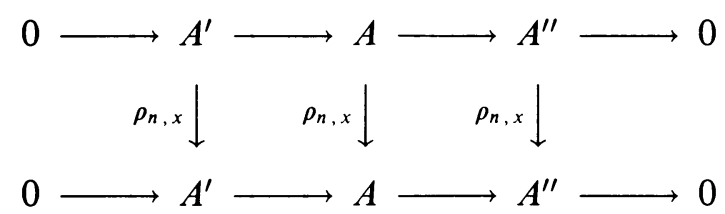

Now our claim follows from the five-lemma.

Lemma 2.7. Let $G$ be a group, $A$ a P-local $\mathbb{Z} G$-module, and $B$ a nilpotent $\mathbb{Z} G$-module. Then the diagonal action of $G$ on each of the following abelian groups is P-local:

$$
A \otimes_{\mathbb{Z}} B, \quad \operatorname{Tor}^{\mathbb{Z}}(A, B), \quad \operatorname{Hom}_{\mathbb{Z}}(B, A), \quad \operatorname{Ext}_{\mathbb{Z}}(B, A) .
$$

Proof. Argue by induction on the nilpotency class of the action of $G$ on $B$, using Lemma 2.6. If this action is trivial, the $\mathbb{Z} G$-module structure on each of the abelian groups listed is induced by that of $A$, i.e. it factors as

$$
\mathbb{Z} G \stackrel{\omega}{\rightarrow} \operatorname{End}(A) \rightarrow \operatorname{End}(C),
$$


where $C$ is any of $A \otimes_{\mathbb{Z}} B, \operatorname{Tor}^{\mathbb{Z}}(A, B), \operatorname{Hom}_{\mathbb{Z}}(B, A), \operatorname{Ext}_{\mathbb{Z}}(B, A)$. Hence, it factors through $\alpha: \mathbb{Z} G \rightarrow(\mathbb{Z} G)_{S}$; cf. (2.5).

Lemma 2.8. Let $N \rightarrow G \rightarrow Q$ be a central group extension and $A$ a P-local $\mathbb{Z} G$-module. Then the induced action of $Q$ on $H_{k}(N ; A)$ and $H^{k}(N ; A)$ is $P$-local for all $k$.

Proof. Since the conjugation action of $G$ on $N$ is trivial, the action of $Q$ on $H_{k}(N ; A)$ is induced by the action of $G$ on $A$, i.e. the following diagram commutes.

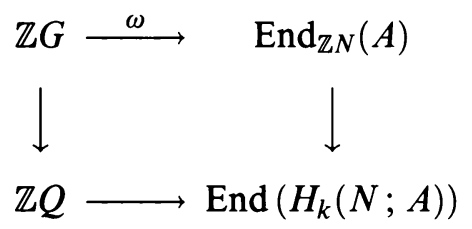

Now use Lemma 2.9 below. The same argument applies to cohomology.

Lemma 2.9. Let $\varphi: G \rightarrow Q$ be a group homomorphism.

(i) If $B$ is a $P$-local $\mathbb{Z} Q$-module, then $B$ is also $P$-local as a $\mathbb{Z} G$-module.

(ii) If $\varphi$ is an epimorphism then the converse in (i) also holds.

Proof. This is straightforward.

In particular, every $P$-local $\mathbb{Z} G$-module is a $\mathbb{Z}_{P}$-module. If the action of $G$ is trivial, then the two notions coincide.

Lemma 2.10. Every nilpotent action of a P-local nilpotent group $G$ on a $\mathbb{Z}_{P^{-}}$ module $A$ is P-local.

Proof. This follows from Remark 2.5; cf. [16].

Lemma 2.11. Let $N \nrightarrow G \rightarrow Q$ be a group extension in which $N$ is P-local and $Q$ is $P$-torsion. Then $G$ is P-local.

Proof. See [3, Theorem 11.5].

Corollary 2.12. Every action of a P-torsion group $G$ on a $\mathbb{Z}_{P}$-module $A$ is P-local.

Proof. A $P$-torsion group $G$ is always $P$-local. Moreover, $A \rtimes G$ is $P$-local by Lemma 2.11. Thus our assertion follows from Remark 2.5.

Corollary 2.13. If the connected $C W$-complex $X$ has a finite P-group as its fundamental group, and $\mathbb{Z}_{P}$-modules as its higher homotopy groups, then $X$ is a P-local space.

Lemma 2.14. Suppose $A$ is a P-local $\mathbb{Z} G$-module. Then every $P^{\prime}$-torsion element of $G$ acts as the identity on $A$.

Proof. If $x$ is $P^{\prime}$-torsion, then $x^{n}=1$ for some $n \in P^{\prime}$, and hence we have the following identity in the ring $\mathbb{Z} G$ :

$$
0=x^{n}-1=(x-1)\left(1+x+x^{2}+\cdots+x^{n-1}\right) .
$$

Since $1+x+x^{2}+\cdots+x^{n-1}$ acts as an isomorphism of $A$, it follows that $x-1$ acts as 0 , i.e. $x a=a$ for every $a \in A$.

Corollary 2.15. If $G$ is a $P^{\prime}$-torsion group, then all $P$-local $\mathbb{Z} G$-modules are trivial $\mathbb{Z} G$-modules. 
We conclude this section by initiating a comparison of the rings $\mathbb{Z}_{P} G$ and $(\mathbb{Z} G)_{S}$, for a $P$-local group $G$. The lemma below follows directly from the definitions.

Lemma 2.16. Let $G$ be any group. Then $(\mathbb{Z} G)_{S}$ is canonically isomorphic to $\mathbb{Z}_{P} G$ if and only if all elements of the form $1+x+\cdots+x^{n-1}, \quad x \in G, n \in P^{\prime}$, are units in $\mathbb{Z}_{P} G$.

On the other hand, we have

Lemma 2.17. Let $f: \mathbb{Z} G \rightarrow R$ be a ring homomorphism. View $R$ as a left $\mathbb{Z} G$ module via $f$. Then $R$ is a P-local $\mathbb{Z} G$-module iff $f\left(1+x+\cdots+x^{n-1}\right)$ is a unit in $R$ for all $x \in G, n \in P^{\prime}$.

Proof. This follows from considering the ring homomorphisms

$$
\mathbb{Z} G \stackrel{f}{\rightarrow} R \stackrel{\mu}{\rightarrow} \operatorname{End}(R)
$$

where $(\mu(r))(s)=r s$, and observing that the inverse image of $\operatorname{Aut}(R)$ under $\mu$ is precisely the group of units of $R$.

Theorem 2.18. Let $G$ be a P-torsion group. Then

(a) $1+x+\cdots+x^{n-1}$ is a unit in $\mathbb{Z}_{P} G$ for all $x \in G, n \in P^{\prime}$.

(b) $(\mathbb{Z} G)_{S} \cong \mathbb{Z}_{P} G$.

Proof. Given $x \in G$, let $C=\langle x\rangle$ be the cyclic subgroup of $G$ generated by $x$. By Corollary $2.12, \mathbb{Z}_{P} C$ is a $P$-local $\mathbb{Z} C$-module via the inclusion $\mathbb{Z} C \rightarrow \mathbb{Z}_{P} C$. Now use Lemma 2.17 to infer (a) and Lemma 2.16 to infer (b).

Alternatively, a proof of Theorem 2.18 can be based on Galois theory, using the ring isomorphism $\phi: \mathbb{Q} C \rightarrow \prod_{d \mid m} \mathbb{Q}\left(\omega^{\frac{m}{d}}\right)$, cf. [25], where $C$ is any cyclic subgroup of $G, m$ the order of $C$, and $\omega=e^{\frac{2 \pi}{m} i}$. We owe this argument to S. Sehgal.

\section{The $P$-localization functor $X \rightarrow X_{P}$}

Theorem 2.1 suggests to regard $P$-local $\mathrm{CW}$-complexes as classifying spaces of $A_{\infty}$-associative $H$-groups which are $P$-local in the sense that their $P^{\prime}$-power maps are self-homotopy equivalences. Due to the special nature of such spaces, we investigate them here in more detail.

$P$-local CW-complexes form a class $\mathscr{D}_{P}$ which fits into an orthogonal pair $\left(\mathscr{S}_{P}, \mathscr{D}_{P}\right)$ in $\mathbf{H o}$ (the pointed homotopy category of connected CW-complexes). Moreover, $\mathscr{D}_{P}$ is the orthogonal complement of a countable collection of maps; see Theorem 3.1. Maps in $\mathscr{S}_{P}$ induce a $P$-equivalence of fundamental groups and isomorphisms in (co-)homology with certain twisted $P$-local coefficients; see Theorem 3.2. Next, we construct an idempotent functor ()$_{P}$ associated to the orthogonal pair $\left(\mathscr{S}_{P}, \mathscr{D}_{P}\right)$ in the sense of $\S 1$. This functor indeed extends $P$-localization of nilpotent spaces, as is shown in $\S 4$.

In the category of groups, the class of $P$-local groups $d_{P}$ is the orthogonal complement of the set

$$
t_{P}=\left\{\mathbb{Z} \stackrel{\cdot n}{\rightarrow} \mathbb{Z}, \quad n \in P^{\prime}\right\} .
$$

Setting $s_{P}=\left(d_{P}\right)^{\perp}$, we obtain an orthogonal pair $\left(s_{P}, d_{P}\right)$ in the category of groups. We refer to homomorphisms in $s_{P}$ as P-equivalences. It is well 
known $[12,22]$ that there is an idempotent monad $\left(()_{P}, l\right)$ associated with the orthogonal pair $\left(s_{P}, d_{P}\right)$, referred to as P-localization of groups. It is also known $[14,24]$ that $\left(()_{P}, l\right)$ induces on nilpotent groups $P$-localization in the classical sense [16]. Moreover, $\left(()_{P}, l\right)$ is readily seen to be the maximal extension of $P$-localization of nilpotent groups, cf. Remark 1.4 above.

For $k \geq 2$, we denote

$$
S_{\tau}^{k}=S^{1} \wedge\left(S^{k-1} \cup \mathrm{pt}\right),
$$

where pt denotes a disjoint base point. Thus $S_{\tau}^{k}$ is a twisted Moore space whose homotopy type is that of $S^{1} \vee S^{k}$, for $k \geq 2$, but their respective suspension-induced co- $H$-structures are not isomorphic, cf. (3.4) below. For convenience, we define $S_{\tau}^{1}=S^{1}$. Let $\rho_{n}: S^{1} \rightarrow S^{1}$ be the standard map of degree $n$, and consider the set

$$
\mathscr{T}_{P}=\left\{\rho_{n}^{k}: S_{\tau}^{k} \rightarrow S_{\tau}^{k}, \quad k \geq 1, n \in P^{\prime}\right\},
$$

where $\rho_{n}^{1}=\rho_{n}$ and $\rho_{n}^{k}=\rho_{n} \wedge$ Id for $k \geq 2$. Then we have

Theorem 3.1. With the above notation, $\left(\mathscr{T}_{P}\right)^{\perp}=\mathscr{D}_{P}$ in Ho.

Proof. By (2.1), there are natural group homomorphisms

$$
\left[S_{\tau}^{k}, X\right] \cong\left[S^{k-1} \cup \mathrm{pt}, \Omega X\right] \cong \pi_{k}(X) \rtimes \pi_{1}(X)
$$

for $k \geq 2$ and every connected space $X$. Moreover, the induced function

$$
\left(\rho_{n}^{k}\right)^{*}:\left[S_{\tau}^{k}, X\right] \rightarrow\left[S_{\tau}^{k}, X\right]
$$

is precisely the $n$th power map for $k \geq 1$. Thus our claim follows from Theorem 2.1.

We conclude that the class $\mathscr{D}_{P}$ is saturated. Maps in $\mathscr{S}_{P}=\left(\mathscr{D}_{P}\right)^{\perp}$ are called $P$-equivalences. Our next goal is to give an explicit description of these. For a group $G$ and a set of primes $P$, we shall write

$$
P[G]=\left(\mathbb{Z}\left[G_{P}\right]\right)_{S}
$$

where $S$ is the multiplicative closure of the elements $1+x+x^{2}+\cdots+x^{n-1}$, $x \in G_{P}, \quad n \in P^{\prime}$, in the ring $\mathbb{Z}\left[G_{P}\right]$; cf. (2.5). Hence, $P[G]$-modules are precisely abelian groups provided with a $P$-local action of $G_{P}$. For a space $X$, we denote, again for simplicity,

$$
P[X]=P\left[\pi_{1}(X)\right] .
$$

With this notation, we have the following characterization of $P$-equivalences.

Theorem 3.2. Let $f: X \rightarrow Y$ be a map between connected spaces. Then $f$ is a $P$-equivalence if and only if $f_{*}: \pi_{1}(X) \rightarrow \pi_{1}(Y)$ is a P-equivalence of groups and

$$
f^{*}: H^{k}(Y ; A) \cong H^{k}(X ; A)
$$

for all $k$ and every (left) $P[Y]$-module $A$ or, equivalently,

$$
f_{*}: H_{k}(X ; P[Y]) \cong H_{k}(Y ; P[Y])
$$

for all $k$. 
Proof. Our argument is analogous to the one sketched in [1, p. 84]. If $f: X \rightarrow$ $Y$ is a $P$-equivalence, then, for each $P$-local group $G, f$ is orthogonal to $K(G, 1)$, and this provides a bijection

$$
f^{*}: \operatorname{Hom}\left(\pi_{1}(Y), G\right) \cong \operatorname{Hom}\left(\pi_{1}(X), G\right) .
$$

This proves the first assertion. The second assertion is proved using a twisted Eilenberg-Mac Lane space [15]: Given a $P[Y]$-module $A$ and an integer $n \geq 1$, set $G=\pi_{1}(Y)_{P}$ and let $G$ act by homeomorphisms on a $K(A, n)$. Consider the space

$$
L_{G}(A, n)=E G \times{ }_{G} K(A, n),
$$

where $E G$ is the universal cover of $K(G, 1)$. If $n \geq 2$, then $L_{G}(A, n)$ has precisely two nonvanishing homotopy groups: $G$ in dimension 1 and $A$ in dimension $n$. Moreover, the induced action of $G$ on $A$ is the given one. If $n=1$, then $L_{G}(A, n)=K(A \rtimes G, 1)$. In any case, by Theorem 2.1 and Remark 2.5, $L_{G}(A, n)$ is $P$-local and hence orthogonal to $f$. This gives a bijection

$$
f^{*}:\left[Y, L_{G}(A, n)\right] \cong\left[X, L_{G}(A, n)\right] \text {, }
$$

which restricts to an isomorphism [15]

$$
f^{*}: H^{n}(Y ; A) \cong H^{n}(X ; A),
$$

as required. The case $n=0$ is trivial. This implies (a).

Now assume that $f_{*}: \pi_{1}(X) \rightarrow \pi_{1}(Y)$ is a $P$-equivalence and that (a) holds. Then, for a map $g: X \rightarrow Z$ with $Z P$-local, there is a unique homomorphism $\varphi: \pi_{1}(Y) \rightarrow \pi_{1}(Z)$ rendering commutative the diagram

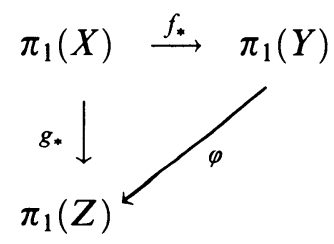

because $f_{*}$ is a $P$-equivalence and $\pi_{1}(Z)$ is $P$-local. Now $\varphi$ can be realized by a map from the two-skeleton of $Y$ to $Z$, whose restriction to the oneskeleton is uniquely determined, up to homotopy, by $\varphi$. Thus, the obstructions to existence and uniqueness, up to homotopy, of a map $g^{\prime}: Y \rightarrow Z$ such that $g^{\prime} f \simeq g$ lie in the cohomology groups

$$
H^{k}\left(Y, X ; \pi_{i}(Z)\right), \quad i \geq 2, \quad k \in\{i+1, i\} .
$$

But these vanish by assumption, because the groups $\pi_{i}(Z), i \geq 2$, are $P[Y]$ modules via $\varphi$, cf. Lemma 2.9. Next, (b) $\Rightarrow$ (a) follows from Lemma 5.3. Finally, (a) $\Rightarrow$ (b) follows from Corollary 7.3.

Now we proceed to prove the existence of an idempotent monad $\left(()_{P}, l\right)$ associated with the orthogonal pair $\left(\mathscr{S}_{P}, \mathscr{D}_{P}\right)$. The argument is a direct application of the technique developed in [12]. 
Theorem 3.3. For every connected space $X$ there is a map $l: X \rightarrow X_{P}$ in $\mathscr{S}_{P}$ with $X_{P}$ in $\mathscr{D}_{P}$.

Proof. For each map $\rho_{n}^{k}: S_{\tau}^{k} \rightarrow S_{\tau}^{k}, \quad k \geq 1, n \in P^{\prime}$, construct the homotopy push-out (double mapping cylinder)

$$
\begin{array}{cc}
S_{\tau}^{k} \stackrel{\rho_{n}^{k}}{\longrightarrow} S_{\tau}^{k} \\
\rho_{n}^{k} \downarrow & \\
S_{\tau}^{k} \underset{t_{n, k}^{2}}{\longrightarrow} & Z_{n, k}
\end{array}
$$

and observe that the maps $t_{n, k}^{i}$ are $P$-equivalences, because the $\rho_{n}^{k}$ are such. Define, by means of (3.10), maps

$$
u_{n, k}: Z_{n, k} \rightarrow S_{\tau}^{k}
$$

such that $u_{n, k} t_{n, k}^{i}=$ Id for all $k \geq 1, n \in P^{\prime}, i=1,2$. Then the maps $u_{n, k}$ are $P$-equivalences as well. We next construct a direct system

$$
X=X_{0} \stackrel{s_{0}}{\longrightarrow} X_{1} \stackrel{s_{1}}{\longrightarrow} X_{2} \stackrel{s_{2}}{\longrightarrow} \cdots
$$

in which each $s_{i}$ is a $P$-equivalence. Assume $X_{i}$ has been constructed for $i \geq 0$. If $X_{i}$ is $P$-local, set $X_{P}=X_{i}$. Otherwise, for each $n \in P^{\prime}$ and each element $z \in \pi_{k}\left(X_{i}\right) \rtimes \pi_{1}\left(X_{i}\right)$ not having an $n$th root, choose a map $\varphi_{z}: S_{\tau}^{k} \rightarrow X_{i}$ representing $z$. For each pair $x, y$ of elements of $\pi_{k}\left(X_{i}\right) \rtimes \pi_{1}\left(X_{i}\right)$ such that $x^{n}=y^{n}, n \in P^{\prime}$, choose a map $\psi_{x, y}: Z_{n, k} \rightarrow X_{i}$ using (3.10), in such a way that $\psi_{x, y} t_{n, k}^{1}$ represents $x$ and $\psi_{x, y} t_{n, k}^{2}$ represents $y$. Define a homotopy push-out

$$
\begin{array}{ccc}
\bigvee_{n, k}\left(\left(\mathrm{~V}_{z} S_{\tau}^{k}\right) \vee\left(\bigvee_{x, y} Z_{n, k}\right)\right) & & \mathrm{V}_{n, k}\left(\left(\mathrm{~V}_{z} S_{\tau}^{k}\right) \vee\left(\mathrm{V}_{x, y} S_{\tau}^{k}\right)\right) \\
\downarrow & & \downarrow \\
X_{i} & \stackrel{s_{i}}{\longrightarrow} & X_{i+1}
\end{array}
$$

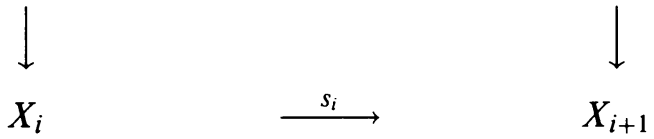

in which the upper arrow is a coproduct of copies of maps $\rho_{n}^{k}$ and $u_{n, k}$ (which is a $P$-equivalence) and the left-hand vertical arrow is defined by means of the maps $\varphi_{z}$ and $\psi_{x, y}$ as chosen above. Therefore, $s_{i}$ is a $P$-equivalence. Let $X_{P}$ be the homotopy direct limit of (3.12), and $l: X \rightarrow X_{P}$ the composite map. It is plain that $l$ is a $P$-equivalence. To check that $X_{P}$ is $P$-local, it suffices to show that $X_{P}$ is orthogonal to each $\rho_{n}^{k}$. But this is ensured by the construction of (3.13), together with the compactness of the spaces $S_{\tau}^{k}$ and $Z_{n, k}$.

Proposition 3.4. For every connected space $X$, the induced homomorphism $l_{*}$ : $\pi_{1}(X) \rightarrow \pi_{1}\left(X_{P}\right)$ is P-localization in the category of groups.

Proof. This is an immediate consequence of Theorem 2.1 and Theorem 3.2.

\section{BEHAVIOUR OF THE FUNCTOR ()$_{P}$ ON NILPOTENT SPACES}

Let $\left(\mathscr{S}_{P}^{\prime}, \mathscr{D}_{P}^{\prime}\right)$ be the orthogonal pair associated with $P$-localization of nilpotent spaces $[7,16]$. In this section we prove 
Theorem 4.1. The orthogonal pair $\left(\mathscr{S}_{P}, \mathscr{D}_{P}\right)$ discussed in $\S 3$ extends $\left(\mathscr{S}_{P}^{\prime}, \mathscr{D}_{P}^{\prime}\right)$, that is, $\mathscr{S}_{P}^{\prime} \subseteq \mathscr{S}_{P}$ and $\mathscr{D}_{P}^{\prime} \subseteq \mathscr{D}_{P}$.

Consequently, for every nilpotent CW-complex $X$ the map $l: X \rightarrow X_{P}$ given by Theorem 3.3 is equal to the $P$-localization of $X$ in the classical sense.

Proof of Theorem 4.1. The fact that the class $\mathscr{D}_{P}^{\prime}$ is contained in $\mathscr{D}_{P}$ has been shown in $\S 2$. We next check that $\mathscr{S}_{P}^{\prime}$ is contained in $\mathscr{S}_{P}$. Let $f: X \rightarrow Y$ be in $\mathscr{S}_{P}^{\prime}$, i.e. the spaces $X, Y$ are nilpotent and

$$
f_{*}: H_{k}\left(X ; \mathbb{Z}_{P}\right) \cong H_{k}\left(Y ; \mathbb{Z}_{P}\right) \text { for all } k \text {. }
$$

Then, as pointed out at the beginning of $\S 3, f_{*}: \pi_{1}(X) \rightarrow \pi_{1}(Y)$ is certainly a $P$-equivalence of groups. Hence, it suffices to prove that

$$
f_{*}: H_{k}(X ; A) \cong H_{k}(Y ; A) \text { for all } k \text { and every } P[Y] \text {-module } A ;
$$

see Theorem 3.2. Fix one such module $A$. Plainly, it suffices to prove (4.2) for maps $f: X \rightarrow Y$ which $P$-localize in the classical sense. In that case, we have a homotopy commutative diagram of universal covers, in which the vertical arrows are $P$-localizations of nilpotent spaces [16]:

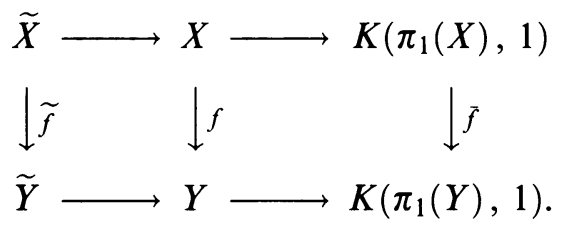

Since the spaces $\tilde{X}, \tilde{Y}$ are simply connected, we have

$$
(\tilde{f})_{*}: H_{k}(\tilde{X} ; A) \cong H_{k}(\tilde{Y} ; A) \text { for all } k \text {, }
$$

because $A$ is in particular a $\mathbb{Z}_{P}$-module. Now we look at the induced homomorphisms

$$
(\bar{f})_{*}: H_{r}\left(\pi_{1}(X) ; H_{s}(\tilde{X} ; A)\right) \rightarrow H_{r}\left(\pi_{1}(Y) ; H_{s}(\tilde{Y} ; A)\right),
$$

which are isomorphisms for all $r, s$, by Lemma 4.2 and Theorem 4.3 below, together with (4.4). Therefore, the morphism of spectral sequences with coefficient module $A$ associated with (4.3) is indeed an isomorphism, which implies (4.2). This completes the argument.

Lemma 4.2. Let $G$ be a group acting by homeomorphisms on a connected $C W$ complex $X$. Assume that the induced action of $G$ on $H_{k}(X ; \mathbb{Z})$ is nilpotent for all $k$. Then, for every $P$-local $\mathbb{Z} G$-module $A$, the diagonal action of $G$ on $H_{k}(X ; A)$ is $P$-local for all $k$ (here $A$ is viewed as a trivial coefficient module in $X)$.

Proof. The case $k=0$ is trivial. For $k \geq 1$, we have a short exact sequence of $\mathbb{Z} G$-modules (with diagonal action)

$$
0 \rightarrow H_{k}(G ; \mathbb{Z}) \otimes_{\mathbb{Z}} A \rightarrow H_{k}(G ; A) \rightarrow \operatorname{Tor}^{\mathbb{Z}}\left(H_{k-1}(G ; \mathbb{Z}), A\right) \rightarrow 0 .
$$

Now Lemma 2.6 and Lemma 2.7 give the desired result.

This applies, of course, to $G=\pi_{1}(Y), X=\widetilde{Y}$ in (4.5). The action of $\pi_{1}(Y)$ on the groups $H_{k}(\widetilde{Y} ; \mathbb{Z})$ is nilpotent because the space $Y$ is nilpotent $[16$, p. 70$]$. 
Theorem 4.3. Let $G$ be a nilpotent group and $l: G \rightarrow G_{P}$ its P-localization. Then, for every $P$-local $\mathbb{Z}\left[G_{P}\right]$-module $A$, the homomorphisms

$$
l_{*}: H_{k}(G ; A) \rightarrow H_{k}\left(G_{P} ; A\right) \text { and } l^{*}: H^{k}\left(G_{P} ; A\right) \rightarrow H^{k}(G ; A)
$$

are isomorphisms for all $k$.

Proof. We shall give the proof only for homology, since it is completely analogous in the case of cohomology. The following argument is partially due to $\mathrm{A}$. Reynol [21].

We argue inductively on the nilpotency class $c$ of $G$. Thus assume first that $G$ is commutative, so that we can consider the group extensions

$$
\begin{gathered}
0 \rightarrow \operatorname{Ker} l \rightarrow G \stackrel{l}{\rightarrow} \operatorname{im} l \rightarrow 0 \\
0 \rightarrow \operatorname{im} l \stackrel{j}{\rightarrow} G_{P} \rightarrow \operatorname{coker} l \rightarrow 0
\end{gathered}
$$

and the corresponding Lyndon-Hochschild-Serre spectral sequences with coefficient module $A$. Note first that the action of $\operatorname{Ker} l$ on $A$ is trivial and, hence, from the fact that $\operatorname{Ker} l$ is $P^{\prime}$-torsion [16] and the Universal Coefficient Theorem it follows that

$$
H_{s}(\operatorname{Ker} l ; A)=0 \quad \text { for all } s \geq 1
$$

and

$$
H_{0}(\operatorname{Ker} l ; A) \cong A \text {. }
$$

Therefore, the spectral sequence associated with (4.6) collapses and gives isomorphisms

$$
l_{*}: H_{k}(G ; A) \cong H_{k}(\mathrm{im} l ; A) \text { for all } k .
$$

Now look at the spectral sequence associated with (4.7). The action of coker $l$ on $H_{s}(\mathrm{im} l ; A)$ is $P$-local by Lemma 2.8 and hence trivial by Corollary 2.15 , because coker $l$ is certainly $P^{\prime}$-torsion. This tells us, again by the Universal Coefficient Theorem, that

$$
H_{r}\left(\operatorname{coker} l ; H_{s}(\operatorname{im} l ; A)\right)=0 \text { for } r \geq 1
$$

and

$$
H_{0}\left(\operatorname{coker} l ; H_{s}(\operatorname{im} l ; A)\right) \cong H_{s}(\operatorname{im} l ; A),
$$

so that we obtain isomorphisms

$$
j_{*}: H_{k}(\operatorname{im} l ; A) \cong H_{k}\left(G_{P} ; A\right) \text { for all } k .
$$

Finally, (4.8) and (4.9) together complete the proof in the case $c=1$. The inductive argument is carried out without any additional difficulty, by using Lemma 2.8 and the diagram of central extensions

$$
\begin{array}{ccccc}
\Gamma^{c-1} G & \mapsto & G & \rightarrow & G / \Gamma^{c-1} G \\
l \downarrow & & l \downarrow & & l \downarrow \\
\left(\Gamma^{c-1} G\right)_{P} & \mapsto & G_{P} & \rightarrow & \left(G / \Gamma^{c-1} G\right)_{P}
\end{array}
$$

where $\Gamma^{k} G, k \geq 0$, denotes terms in the lower central series of $G$. 
Remark 4.4. Theorem 4.3 actually extends Theorem 4.14 in [16, p. 40], since every nilpotent action of a nilpotent $P$-local group on a $\mathbb{Z}_{P}$-module is $P$-local (Lemma 2.10).

We also record a useful corollary of Theorem 4.3, applications of which have been given in [21].

Corollary 4.5. Let $G$ be a nilpotent group and $l: G \rightarrow G_{P}$ its P-localization. Assume that $G_{P}$ acts on a $\mathbb{Z}_{P}$-module $A$ through $\omega: G_{P} \rightarrow \operatorname{Aut}(A)$ in such a way that $\operatorname{im} \omega$ is a torsion group of finite exponent. Then

$$
l_{*}: H_{k}(G ; A) \cong H_{k}\left(G_{P} ; A\right)
$$

and

$$
l^{*}: H^{k}\left(G_{P} ; A\right) \cong H^{k}(G ; A) \text { for all } k \text {. }
$$

Proof. Set $Q=\operatorname{im} \omega$. Then $Q$ is a finite $P$-group because it is an epimorphic image of a $P$-local group. Hence, by Corollary 2.12 , the action of $Q$ on $A$ is $P$-local and, by Lemma 2.9 , so is the action of $G_{P}$ on $A$. Now Theorem 4.3 applies.

Note that the hypothesis concerning the action $\omega$ is essential. For otherwise we have the explicit counterexample $G=\mathbb{Z}$ and $A=\mathbb{Q}[\mathbb{Q}$; see [10].

\section{LOCALIZING WITH RESPECT TO HOMOLOGY WITH TWISTED COEFFICIENTS}

In this section and the two next ones we consider our $P$-localization functor from the homology point of view. We show that it is a particular output of a certain rich source of idempotent monads in Ho. In fact, Bousfield's localization with respect to homology [4] can be paralleled for homology with twisted coefficients, provided we work in the appropriate categories. The resulting localizing functors can be "spliced" to produce localizing functors in Ho, some of which extend the familiar $P$-localization of nilpotent spaces. In this section, we explain the procedure of localizing with respect to homology with twisted coefficients. Splicing will be described in $\S 6$.

For a group $G$, denote by $\operatorname{Ho}(G)$ the category whose objects are pairs $(X, \varphi)$, where $X$ is a connected space and $\varphi: \pi_{1}(X) \rightarrow G$ is a group homomorphism. Morphisms from $(X, \varphi)$ to $(Y, \psi)$ are (pointed homotopy classes of) maps $f: X \rightarrow Y$ such that $\varphi=\psi f_{*}$, where $f_{*}: \pi_{1}(X) \rightarrow \pi_{1}(Y)$ is the homomorphism induced by $f$.

Theorem 5.1. Let $G$ be a group and $A$ a (right) $\mathbb{Z} G$-module. Then there is an idempotent monad $(E, \eta)$ in the category $\mathbf{H o}(G)$ whose class of E-equivalences consists precisely of all $H_{*}(;$ A)-equivalences.

We base our proof of Theorem 5.1 on the existence result for localizing functors given in [12]. For the convenience of the reader, it is reproduced as Theorem 5.2 below, in a form adapted to our present purposes.

Theorem 5.2. Suppose that a class $\mathscr{S}$ of morphisms in $\mathbf{H o}(G)$ satisfies conditions $(\mathrm{C} 1)$ to $(\mathrm{C} 6)$ below. Then $\mathscr{S}$ is saturated and $\mathrm{Ho}(G)$ admits a localizing functor whose class of equivalences is $\mathscr{S}$.

(C1) $\mathscr{S}$ contains all isomorphisms in $\mathrm{Ho}(G)$.

(C2) If the composite $g f$ of two morphisms is defined and any two of $f, g$, $g f$ are in $\mathscr{S}$, then the third is also in $\mathscr{S}$. 
(C3) The coproduct of a set of morphisms in $\mathscr{S}$ is in $\mathscr{S}$.

(C4) There is a set $\mathscr{S}_{0} \subseteq \mathscr{S}$ such that $\left(\mathscr{S}_{0}\right)^{\perp}=\mathscr{S}^{\perp}$.

(C5) For every diagram $C \stackrel{f}{\longleftarrow} A \stackrel{s}{\longrightarrow} B$ with $s \in \mathscr{S}$ there is a weak push-out

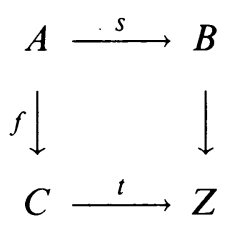

with $t \in \mathscr{S}$.

(C6) For every ordinal $c$ and every directed system $F: c \rightarrow \mathbf{H o}(G)$ with $s_{i}: F(0) \rightarrow F(i)$ in $\mathscr{S}$ for all $i<c$, there is a weak colimit $T$ such that the morphism $s_{c}: F(0) \rightarrow T$ is in $\mathscr{S}$.

Now we proceed to verify these hypotheses in our case.

Proof of Theorem 5.1. Let $\mathscr{S}$ be the class of $H_{*}(; A)$-equivalences in $\mathrm{Ho}(G)$. Conditions (C1), (C2) and (C3) are obviously satisfied. Let $c$ be the smallest infinite ordinal whose cardinality is bigger than the cardinality of $A$. The collection of spaces $X$ with at most $c$ cells forms a proper set of homotopy types. Choose a representing space in each of these homotopy types, and let $\mathscr{S}_{0}$ consist of all morphisms $f:(X, \varphi) \rightarrow(Y, \psi)$ in $\mathscr{S}$ where $X$ and $Y$ are such representing spaces. Then Lemma 11.3 in [4] can be adapted to also incorporate twisted coefficients. This proves that $\left(\mathscr{S}_{0}\right)^{\perp}=\mathscr{S}^{\perp}$. Hence, condition (C4) is satisfied. In (C5) and (C6) we can simply take the corresponding homotopy colimits, together with the obvious group homomorphisms.

In general, the construction of $\eta_{X}: X \rightarrow E X$ for a given space $X$ will require a homotopy direct limit of length up to the ordinal $c$; see $[4,11]$.

The next lemmas are the key to interrelate the functors given by Theorem 5.1. Note that, if $(X, \varphi)$ is an object of $\mathrm{Ho}(G)$, then each ring homomorphism $\alpha: \mathbb{Z} G \rightarrow R$ allows us to regard $R$ as a twisted coefficient module in $X$.

Lemma 5.3. Let $G$ be a group and $f:(X, \varphi) \rightarrow(Y, \psi)$ a morphism in $\mathbf{H o}(G)$. Let $\alpha: \mathbb{Z} G \rightarrow R$ be a ring homomorphism. If $f$ is an $H_{*}(; R)$-equivalence, then

(i) $f$ is an $H_{*}($; A)-equivalence for every right $R$-module $A$; and

(ii) $f$ is an $H^{*}(; B)$-equivalence for every left $R$-module $B$.

Proof. We can assume, without loss of generality, that $X$ is a subcomplex of $Y$ and that $f$ is the corresponding inclusion. Let $q: \widetilde{Y} \rightarrow Y$ be the universal cover of $Y$ and set $\widehat{X}=q^{-1}(X), \quad \pi=\pi_{1}(Y)$. Let $C_{*}=C_{*}(\tilde{Y}, \widehat{X})$ denote the associated cellular chain complex. Then the hypothesis,

$$
0=H_{k}(Y, X ; R)=H_{k}\left(R \otimes_{\mathbb{Z} \pi} C_{*}\right) \text { for all } k,
$$

tells us precisely that $R \otimes_{\mathbb{Z} \pi} C_{*}$ is a free resolution of 0 as an $R$-module. Consequently,

$$
H_{k}(Y, X ; A) \cong H_{k}\left(A \otimes_{R} R \otimes_{\mathbb{Z} \pi} C_{*}\right)=\operatorname{Tor}_{k}^{R}(A, 0)=0
$$


and

$$
\begin{aligned}
H^{k}(Y, X ; B) & \cong H^{k} \operatorname{Hom}_{\mathbb{Z} \pi}\left(C_{*}, \operatorname{Hom}_{R}(R, B)\right) \\
& \cong H^{k} \operatorname{Hom}_{R}\left(R \otimes_{\mathbb{Z} \pi} C_{*}, B\right) \\
& =\operatorname{Ext}_{R}^{k}(0, B)=0 \text { for all } k . \quad \square
\end{aligned}
$$

Let $e: \mathbb{Z} \rightarrow \mathbb{Z}_{P}$ denote the inclusion, and $e_{n}$ denote the composite

$$
\mathbb{Z} \stackrel{\cdot n}{\longrightarrow} \mathbb{Z} \stackrel{e}{\longrightarrow} \mathbb{Z}_{P}
$$

Given a $\mathbb{Z}\left[\mathbb{Z}_{P}\right]$-module $A$, we shall denote by $e_{n}^{*} A$ the $\mathbb{Z}[\mathbb{Z}]$-module defined via (5.1).

With the notation of $\S 3$, we can consider in $\mathbf{H o}\left(\mathbb{Z}_{P}\right)$ the morphisms

$$
\rho_{n}^{k}:\left(S_{\tau}^{k}, e_{n}\right) \rightarrow\left(S_{\tau}^{k}, e\right), \quad k \geq 1, n \in P^{\prime} .
$$

Lemma 5.4. (i) $\rho_{n}^{k}$ is an $H_{*}(; P[\mathbb{Z}])$-equivalence for all $k \geq 1, n \in P^{\prime}$.

(ii) $H_{1}\left(S_{\tau}^{k} ; A\right)=0$ for all $k \geq 1$, where $A$ is any one of the following coefficient modules $\left(n \in P^{\prime}\right)$ :

$$
\mathbb{Z}\left[\mathbb{Z}_{P}\right], \quad e_{n}^{*} \mathbb{Z}\left[\mathbb{Z}_{P}\right], \quad \mathbb{Z}_{P}\left[\mathbb{Z}_{P}\right], \quad e_{n}^{*} \mathbb{Z}_{P}\left[\mathbb{Z}_{P}\right], \quad P[\mathbb{Z}], \quad e_{n}^{*} P[\mathbb{Z}] .
$$

Proof. Let $x$ be a generator of $\pi_{1}\left(S_{\tau}^{k}\right)$. The cellular chain complex of the universal cover of $S_{\tau}^{k}$ consists of a copy of $\mathbb{Z}[\mathbb{Z}]$ in dimensions 0,1 and $k$. Therefore, the homomorphisms

$$
\left(\rho_{n}^{k}\right)_{*}: H_{i}\left(S_{\tau}^{k} ; e_{n}^{*} P[\mathbb{Z}]\right) \longrightarrow H_{i}\left(S_{\tau}^{k} ; P[\mathbb{Z}]\right)
$$

are induced by the chain map

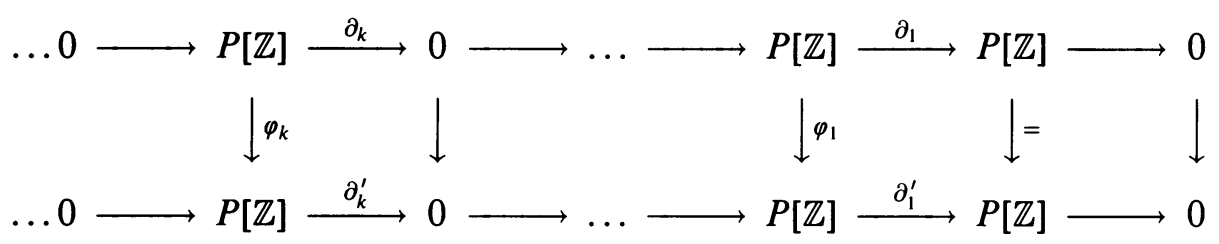

where $\partial_{1}=1-x^{n}, \quad \partial_{1}^{\prime}=1-x, \quad \partial_{k}=\partial_{k}^{\prime}=0($ even if $k=2)$, and

$$
\varphi_{1}=\varphi_{k}=1+x+x^{2}+\cdots+x^{n-1} \text {. }
$$

Therefore, the above chain map is a chain isomorphism and (5.3) is an isomorphism for all $i$, as claimed. To prove (ii), consider the chain map above with coefficient module $A$. First of all, $\partial_{1}$ and $\partial_{1}^{\prime}$ are monomorphisms with any of the first four coefficient systems listed, because $\mathbb{Z}\left[\mathbb{Z}_{P}\right]$ and $\mathbb{Z}_{P}\left[\mathbb{Z}_{P}\right]$ contain no zero divisors. For the remaining two systems, the claim follows because $P[\mathbb{Z}]$ is flat as a $\mathbb{Z}\left[\mathbb{Z}_{P}\right]$-module.

\section{SPlicing localization FUNCTORS}

In this section we show how localization functors on different categories $\mathbf{H o}(G)$ can be spliced to yield localization functors on Ho. The main abstract result is Theorem 6.2. In conjunction with the ordering of orthogonal pairs, as in Remark 1.4, it serves us as a pivotal point to access and compare various idempotent functors on Ho. 
The basic ingredient in Theorem 6.2 is a pair of matching functors. To explain this, suppose given

(i) a localization functor $L$ on the category of groups, with associated orthogonal pair $(\mathscr{S}, \mathscr{D})$;

(ii) a functor $F: \mathbb{Z} \mathscr{D} \rightarrow \mathscr{R}$ together with a natural transformation $T$ : Id $\rightarrow F$. Here $\mathscr{R}$ denotes the category of rings, and $\mathbb{Z} \mathscr{D}$ denotes the category of integral group rings of groups in $\mathscr{D}$ (and ring homomorphisms induced by group homomorphisms).

To a connected CW-complex $X$, we apply the following process: Associate to $X$ the object $\left(X, \varphi_{X}\right)$ in $\mathbf{H o}\left(L \pi_{1}(X)\right)$, where $\varphi_{X}: \pi_{1}(X) \rightarrow L \pi_{1}(X)$ is the localizing homomorphism. By Theorem 5.1, the object $\left(X, \varphi_{X}\right)$ has an $H_{*}\left(; F \mathbb{Z}\left[L \pi_{1}(X)\right]\right)$-localization in the category $\mathbf{H o}\left(L \pi_{1}(X)\right)$. Denote it by $\left(X_{L}^{F}, \psi_{X}\right), \psi_{X}: \pi_{1}\left(X_{L}^{F}\right) \rightarrow L \pi_{1}(X)$. Now forget the twist in this object, so as to obtain again a space $X_{L}^{F}$ in Ho. Schematically,

$$
\begin{array}{cccc} 
& \text { Ho }\left(L \pi_{1}(X)\right):\left(X, \varphi_{X}\right) & & \\
& & & \\
& & & \\
\text { Ho: } & X & \left.X_{L}^{F}, \psi_{X}\right) & \\
\longrightarrow & X_{L}^{F}
\end{array}
$$

Definition 6.1. We say that the functors $F$ and $L$ match if the induced homomorphism $\left(\eta_{X}\right)_{*}: \pi_{1}(X) \rightarrow \pi_{1}\left(X_{L}^{F}\right)$ is an $L$-equivalence for every space $X$.

Theorem 6.2 ("Splicing theorem"). For matching functors $F$ and $L$ the association $X \rightarrow X_{L}^{F}$ determines an idempotent monad on Ho with natural transformation $\eta$.

Proof. First we check functoriality. Thus start with a map $f: X \rightarrow Y$ and let $f_{*}: \pi_{1}(X) \rightarrow \pi_{1}(Y)$ be the induced homomorphism of fundamental groups. Then the morphism in $\mathbf{H o}\left(L \pi_{1}(Y)\right)$

$$
\left(X,\left(L f_{*}\right) \varphi_{X}\right) \stackrel{\eta_{X}}{\longrightarrow}\left(X_{L}^{F},\left(L f_{*}\right) \psi_{X}\right)
$$

is an $H_{*}\left(; F \mathbb{Z}\left[L \pi_{1}(Y)\right]\right)$-equivalence, by Lemma 5.3. Hence, there is a unique morphism $f^{\prime}$ in $\mathbf{H o}\left(L \pi_{1}(Y)\right)$ rendering commutative the diagram

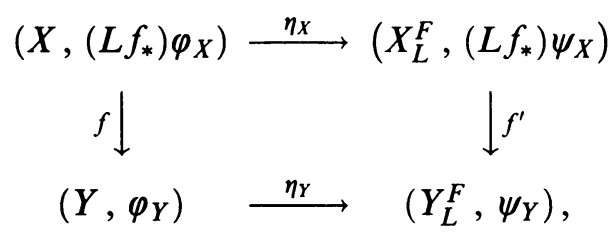

which gives a commutative diagram in $\mathbf{H o}$

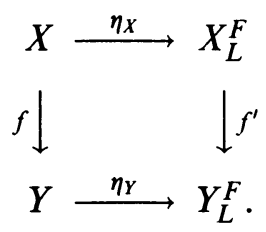

Moreover, if $f^{\prime \prime}: X_{L}^{F} \rightarrow Y_{L}^{F}$ also renders (6.3) commutative, then

$$
\psi_{Y}\left(f^{\prime \prime}\right)_{*}\left(\eta_{X}\right)_{*}=\psi_{Y}\left(\eta_{Y}\right)_{*} f_{*}=\psi_{Y}\left(f^{\prime}\right)_{*}\left(\eta_{X}\right)_{*}=\left(L f_{*}\right) \psi_{X}\left(\eta_{X}\right)_{*},
$$


and, since $\left(\eta_{X}\right)_{*}$ is assumed to be an $L$-equivalence, it follows that $\psi_{Y}\left(f^{\prime \prime}\right)_{*}=$ $\left(L f_{*}\right) \psi_{X}$. Thus $f^{\prime \prime}$ is also a morphism in $\mathbf{H o}\left(L \pi_{1}(Y)\right)$ and hence $f^{\prime \prime} \simeq f^{\prime}$. Functoriality follows. Next we check idempotence. Since $L \pi_{1}\left(X_{L}^{F}\right) \cong L \pi_{1}(X)$ by assumption, the homomorphism $\varphi_{X_{L}^{F}}$ coincides with $\psi_{X}$. Therefore, the object $\left(X_{L}^{F}, \varphi_{X_{L}^{F}}\right)$ is already $H_{*}\left(; F \mathbb{Z}\left[L \pi_{1}(X)\right]\right)$-local, and it follows that the map $\eta_{X_{L}^{F}}=\left(\eta_{X}\right)^{\prime}: X_{L}^{F} \rightarrow\left(X_{L}^{F}\right)_{L}^{F}$ is a homotopy equivalence.

We say that an idempotent monad $(E, \eta)$ in $\mathbf{H o}$ is spliceable if it can be obtained from a pair of matching functors $F, L$ by means of (6.1).

Theorem 6.3. The P-localization monad $\left(()_{P}, l\right)$ described in $\S 3$ is spliceable. Proof. Choose $L$ to be $P$-localization of groups and set $F \mathbb{Z} G=(\mathbb{Z} G)_{S}$; cf. (2.5). Thus, for a $C W$-complex $X, \quad F \mathbb{Z}\left[L \pi_{1}(X)\right]=P[X]$; cf. (3.6). Next we show that the functors $F$ and $L$ match, and that the resulting monad is precisely $\left(()_{P}, l\right)$. Given a space $X$, consider the morphism

$$
l:\left(X, \varphi_{X}\right) \rightarrow\left(X_{P}, \mathrm{Id}\right)
$$

in $\mathbf{H o}\left(\pi_{1}(X)_{P}\right)$. We check that $(6.4)$ is $H_{*}(; P[X])$-localization, so that $X_{L}^{F} \simeq$ $X_{P}$ and $\eta_{X}=l$, as claimed.

First we see that $\left(X_{P}, \mathrm{Id}\right)$ is an $H_{*}(; P[X])$-local object. Thus assume given an $H_{*}(; P[X])$-equivalence $s:(A, u) \rightarrow(B, v)$ and a morphism $f:(A, u) \rightarrow$ $\left(X_{P}, \mathrm{Id}\right)$. Since the groups $\pi_{i}\left(X_{P}\right), i \geq 2$, are $P[X]$-modules, the cohomology groups $H^{k}\left(B, A ; \pi_{i}\left(X_{P}\right)\right)$ vanish for $i \geq 2$, by Lemma 5.3. Now existence and uniqueness of a morphism $g:(B, v) \rightarrow\left(X_{P}\right.$, Id) satisfying $g s=f$ follow by obstruction theory, as in (3.9).

Secondly, we check that $(6.4)$ is an $H_{*}(; P[X])$-equivalence. Let $(C, \omega)$ be an $H_{*}(; P[X])$-local object in $\mathbf{H o}\left(\pi_{1}(X)_{P}\right)$, and assume given a morphism $f$ : $\left(X, \varphi_{X}\right) \rightarrow(C, \omega)$. By Lemma 5.3 and Lemma 5.4, the morphisms $\rho_{n}^{k}: S_{\tau}^{k} \rightarrow$ $S_{\tau}^{k}$ introduced in (3.3) are $H_{*}(; P[X])$-equivalences via any homomorphism $\mathbb{Z} \rightarrow \pi_{1}(X)_{P}$. It follows that $C$ is a $P$-local space, by Theorem 3.1. Since $l: X \rightarrow X_{P} P$-localizes in Ho, existence and uniqueness of a morphism $g$ : $\left(X_{P}, \mathrm{Id}\right) \rightarrow(C, \omega)$ satisfying $g l \simeq f$ is straightforward.

Example 6.4. The class $\mathscr{D}$ of connected spaces whose higher homotopy groups are $P$-local satisfies $\mathscr{D}=\mathscr{T}^{\perp}$ in Ho, where

$$
\mathscr{T}=\left\{\rho_{n}: S^{k} \rightarrow S^{k}, k \geq 2, n \in P^{\prime}\right\},
$$

$\rho_{n}$ denoting the map of degree $n$. Using the same arguments as in Theorem 3.2, one sees that the class $\mathscr{S}=\mathscr{D}^{\perp}$ consists of those maps $f: X \rightarrow Y$ inducing an isomorphism of fundamental groups and being $H^{*}(; A)$-equivalences for every $\mathbb{Z}_{P}\left[\pi_{1}(Y)\right]$-module $A$. Paralleling the arguments of $\S 3$, an idempotent monad $(E, \eta)$ associated with the orthogonal pair $(\mathscr{S}, \mathscr{D})$ can be obtained. We claim that this monad is spliceable. In the setting of Theorem 6.2 , choose $L$ to be the identity and $F \mathbb{Z} G=\mathbb{Z}_{P} \otimes \mathbb{Z} G=\mathbb{Z}_{P} G$. These functors match, and the resulting monad is $(E, \eta)$. The proof is completely analogous to the one given in Example 6.3.

The orthogonal pair generated by $\left\{\rho_{n}: S^{k} \rightarrow S^{k}, k \geq 1, n \in P^{\prime}\right\}$ does not admit a localization functor; see [10]. 
Example 6.5. Given an arbitrary ring $R$, viewed as a trivial coefficient module, the $H_{*}(; R)$-localization functor of $[1,4]$ is spliceable: Choose $L$ to be $L G=\{1\}$ for every group $G$, and $F \mathbb{Z} G=R$.

Example 6.6. For any ring $R$, let $L$ be the $H R$-localization functor on the category of groups [4], and let $F$ be any functor such that $F \mathbb{Z}=R$. Then $L$ and $F$ match because every $H_{*}(; F \mathbb{Z} G)$-equivalence is also an $H_{*}(; R)$-equivalence, which induces an $L$-equivalence of fundamental groups, cf. [4]. If $F \mathbb{Z} G=R$ for every $G$ then the resulting functor is again $H_{*}(; R)$-localization.

We close this section by displaying the ordering relations between some of the spliceable localizing functors explicitly described above; see Remark 1.3. Thus, there is a natural transformation $E \rightarrow E^{\prime}$ if the class of $E$-equivalences is contained in the class of $E^{\prime}$-equivalences.

We denote by ()$_{P}$ both $P$-localization of groups and spaces, and by $E_{P}$ both $H \mathbb{Z}_{P}$-localization of groups and $H_{*}\left(; \mathbb{Z}_{P}\right)$-localization of spaces. In the following diagram, we represent each functor by a matching pair $L, F$ defining it. The arrows are natural transformations.

$$
\text { (A) } \quad L=\mathrm{Id}, F \mathbb{Z} G=\mathbb{Z}_{P} G
$$

(B) $L=()_{P}, F \mathbb{Z} G=(\mathbb{Z} G)_{S}$

(D) $\quad L=E_{P}, \quad F \mathbb{Z} G=(\mathbb{Z} G)_{S}$

(E) $\quad L=E_{P}, F \mathbb{Z} G=\mathbb{Z}_{P}$.

Here $(B)$ is the $P$-localization functor, and $(E)$ is the $H_{*}\left(; \mathbb{Z}_{P}\right)$-localization functor.

Proposition 6.7. The $H_{*}\left(; \mathbb{Z}_{P}\right)$-localization is the minimal extension of $P$ localization of nilpotent spaces.

Proof. The orthogonal complement of all $P$-local nilpotent spaces is contained in the class of all $H_{*}\left(; \mathbb{Z}_{P}\right)$-equivalences, because the spaces $K\left(\mathbb{Z}_{P}, n\right), \quad n \geq$ 1 , are nilpotent and $P$-local. On the other hand, $H^{*}\left(; \mathbb{Z}_{P}\right)$-equivalences coincide with $H_{*}\left(; \mathbb{Z}_{P}\right)$-equivalences and their localization extends $P$-localization of nilpotent spaces. Now our claim follows from Proposition 2.3 in [12].

We conjecture that the functor ()$_{P}$ is the maximal extension of the $P$ localization of nilpotent spaces. We know that the functor $(C)$ in the diagram above does not extend $P$-localization of nilpotent spaces (nor does any functor above it) because an $H_{*}\left(; \mathbb{Z}_{P}\right)$-equivalence between nilpotent spaces need 
not be an $H_{*}(; A)$-equivalence for $A$ an arbitrary coefficient module whose underlying abelian group is $P$-local [10].

7. THE CLASS OF EQUIVALENCES OF A SPLICEABLE LOCALIZATION FUNCTOR

Let $L$ and $F$ be a matching pair of functors in the sense of $\S 6$, and let $(E, \eta)$ be the associated idempotent monad in Ho, cf. Theorem 6.2.

Lemma 7.1. If $G$ is an L-local group, then $K(G, 1)_{L}^{F} \simeq K(G, 1)$.

Proof. It suffices to show that the object $(K(G, 1)$, Id $)$ is $H_{*}(; F \mathbb{Z} G)$-local in $\mathbf{H o}(G)$. But $(K(G, 1), \mathrm{Id})$ is indeed orthogonal to every morphism in $\operatorname{Ho}(G)$.

Theorem 7.2. A map $f: X \rightarrow Y$ in $\mathbf{H o}$ is an E-equivalence if and only if $f_{*}: \pi_{1}(X) \rightarrow \pi_{1}(Y)$ is an L-equivalence and $f$ is an $H_{*}\left(; F \mathbb{Z}\left[L \pi_{1}(Y)\right]\right)$ equivalence.

Proof. If $f$ is an $E$-equivalence, then, for every $L$-local group $G, K(G, 1)$ is $E$-local by Lemma 7.1 and hence orthogonal to $f$. This proves the first claim. Now, in the diagram (6.2), $f^{\prime}:\left(X_{L}^{F},\left(L f_{*}\right) \psi_{X}\right) \rightarrow\left(Y_{L}^{F}, \psi_{Y}\right)$ is an isomorphism by assumption and the horizontal arrows are $H_{*}\left(; F \mathbb{Z}\left[L \pi_{1}(Y)\right]\right)$-equivalences, so that $f$ is also an $H_{*}\left(; F \mathbb{Z}\left[L \pi_{1}(Y)\right]\right)$-equivalence. Conversely, if $f_{*}$ : $\pi_{1}(X) \rightarrow \pi_{1}(Y)$ is an $L$-equivalence, then the localization diagram in Ho

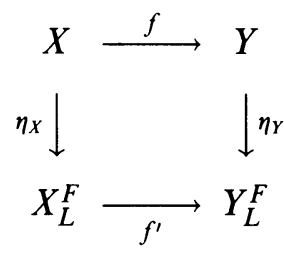

corresponds to a localization diagram in $\mathbf{H o}\left(L \pi_{1}(Y)\right)$. But then $f^{\prime}$ is a homotopy equivalence because $f$ is an $H_{*}\left(; F \mathbb{Z}\left[L \pi_{1}(Y)\right]\right)$-equivalence.

For the spliceable functor ()$_{P}$ we have an independent characterization of its equivalences in terms of cohomology (see Theorem 3.2). Both descriptions together yield

Corollary 7.3. Let $f: X \rightarrow Y$ be a map in Ho inducing a P-equivalence of fundamental groups. Then the following are equivalent:

(i) $f$ is an $H_{*}(; P[Y])$-equivalence.

(ii) $f$ is an $H_{*}($; A)-equivalence for every right $P[Y]$-module $A$.

(iii) $f$ is an $H^{*}(; B)$-equivalence for every left $P[Y]$-module $B$.

\section{Some SPECial features of $P$-LOCAlization}

In this section we consider the connections between the functor ()$_{P}$ discussed in $\S 3$, the $H_{*}\left(; \mathbb{Z}_{P}\right)$-localization functor $E_{P}$, and the $\mathbb{Z}_{P}$-completion functor $\left(\mathbb{Z}_{P}\right)_{\infty}$ of Bousfield-Kan [7]. All spaces $X$ are assumed to be connected.

From [7, II, 2.8] and the results of $\S 6$, we know that for every space $X$ there is a homotopy commutative diagram 


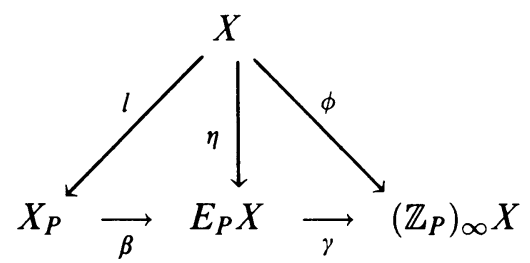

where $l, \eta$ are the respective localization maps, and $\phi$ is the $\mathbb{Z}_{P}$-completion map. Moreover, $\gamma$ is a homotopy equivalence if and only if the space $X$ is

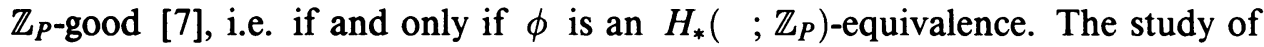
$\mathbb{Z}_{P}$-good spaces continue to be a matter of current interest [6].

If the space $X$ is nilpotent, then $X$ is $\mathbb{Z}_{P}$-good and the maps $\beta, \gamma$ in (8.1) are homotopy equivalences. We provide the following generalization:

Proposition 8.1. If the space $X_{P}$ is nilpotent, then $X$ is $\mathbb{Z}_{P-\text { good }}$ and the maps $\beta, \gamma$ in (8.1) are homotopy equivalences.

Proof. Every nilpotent $P$-local space is $H_{*}\left(; \mathbb{Z}_{P}\right)$-local. Thus, under our assumption, $l: X \rightarrow X_{P}$ is an $H_{*}\left(; \mathbb{Z}_{P}\right)$-equivalence into an $H_{*}\left(; \mathbb{Z}_{P}\right)$-local space and hence $l \simeq \eta$. Secondly, $l$ induces a homotopy equivalence

$$
\left(\mathbb{Z}_{P}\right)_{\infty} X \simeq\left(\mathbb{Z}_{P}\right)_{\infty} X_{P},
$$

and plainly $\left(\mathbb{Z}_{P}\right)_{\infty} X_{P} \simeq X_{P}$, because $X_{P}$ is nilpotent $P$-local. It follows that $\gamma \beta$ is a homotopy equivalence, and hence so is $\gamma$.

The nilpotence of $X_{P}$ can be checked in practice in some cases. For example, it is guaranteed whenever $\pi_{1}(X)_{P}=\{1\}$. Note that spaces such that $\pi_{1}(X)_{P}=$ \{1\} satisfy also $H_{1}\left(X ; \mathbb{Z}_{P}\right)=0$ and hence they are indeed known to be $\mathbb{Z}_{P}$-good [7, VII, 3.2].

Example 8.2. Let $P=\{3\}$ and $\Sigma_{3}$ denote the symmetric group on three elements. Then $\left(\Sigma_{3}\right)_{3}=\{1\}$ and, therefore,

$$
K\left(\Sigma_{3}, 1\right)_{3} \simeq E_{3} K\left(\Sigma_{3}, 1\right) \simeq\left(\mathbb{Z}_{3}\right)_{\infty} K\left(\Sigma_{3}, 1\right),
$$

which is a simply-connected space with nontrivial homotopy [7, VII, 4.4].

This last example turns out to be a particular case of a more general fact (Theorem 8.4 below). We are indebted to G. Mislin for some key ideas in the next proposition.

Proposition 8.3. Let $X$ be a space whose homotopy groups $\pi_{i}(X)$ are finite for $i \geq 1$, and let $P$ consist of a single prime $p$. Then

(i) The homotopy groups $\pi_{i}\left(\left(\mathbb{Z}_{P}\right)_{\infty} X\right)$ are finite p-groups for $i \geq 1$.

(ii) The space $\left(\mathbb{Z}_{P}\right)_{\infty} X$ is nilpotent.

(iii) The $\mathbb{Z}_{P}$-completion map $\phi: X \rightarrow\left(\mathbb{Z}_{P}\right)_{\infty} X$ is an $H_{*}(; A)$-equivalence for every (twisted) coefficient module $A$ whose underlying abelian group is a $\mathbb{Z}_{P}$-module.

(iv) The kernel $N$ of $\phi_{*}: \pi_{1}(X) \rightarrow \pi_{1}\left(\left(\mathbb{Z}_{P}\right)_{\infty} X\right)$ satisfies $N_{P}=\{1\}$.

Proof. Statement (i) is proved in [7, VII, 4.3]. Statement (ii) follows because every action of a finite $p$-group on another finite $p$-group is nilpotent. Now let $F$ be the homotopy fibre of $\phi$. The homotopy groups $\pi_{i}(F)$ are finite for 
$i \geq 1$, and, hence, so are the homology groups $H_{i}(F ; \mathbb{Z}), \quad i \geq 1$. It follows that any action of $\pi_{1}\left(\left(\mathbb{Z}_{P}\right)_{\infty} X\right)$ on the groups $H_{i}\left(F ; \mathbb{Z}_{P}\right)$ must be nilpotent. Hence, we can apply the spectral sequence comparison theorem [17] to the diagram

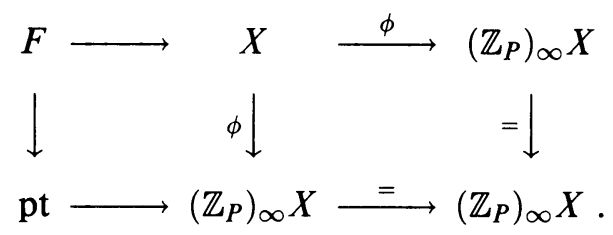

Since $X$ is $\mathbb{Z}_{P}$-good [7, VII, 4.3], $\phi$ is an $H_{*}\left(; \mathbb{Z}_{P}\right)$-equivalence, implying that $H_{i}\left(F ; \mathbb{Z}_{P}\right)=0$, for $i \geq 1$. Now, given a $\mathbb{Z}_{P}$-module $A$ with an action of $\pi_{1}\left(\left(\mathbb{Z}_{P}\right)_{\infty} X\right)$, the groups $H_{i}(F ; A), \quad i \geq 1$, vanish because the action of $\pi_{1}(F)$ on $A$ via $\phi$ is trivial. It follows from the top fibration in (8.3) that

$$
\phi_{*}: H_{i}(X ; A) \cong H_{i}\left(\left(\mathbb{Z}_{P}\right)_{\infty} X ; A\right) \text { for all } i,
$$

thus proving (iii). To prove (iv), observe that $N=\operatorname{Ker}\left(\phi_{*}\right)$ is an epimorphic image of $\pi_{1}(F)$ and, since $P$-localization preserves epimorphisms [22], it suffices to show that $\pi_{1}(F)_{P}=\{1\}$. By Corollary 2.3 in [9],

$$
\pi_{1}(F)_{P} \cong \pi_{1}\left(\left(\mathbb{Z}_{P}\right)_{\infty} F\right) \text {. }
$$

and $\pi_{1}\left(\left(\mathbb{Z}_{P}\right)_{\infty} F\right)$ vanishes because $H_{1}\left(F ; \mathbb{Z}_{P}\right)=0$.

Theorem 8.4. Let $X$ be a space whose homotopy groups $\pi_{i}(X)$ are finite for $i \geq 1$, and $P$ consist of a single prime $p$. Then $X_{P}$ is nilpotent and the maps $\beta, \gamma$ in (8.1) are homotopy equivalences.

Proof. From (iii) and (iv) in Proposition 8.3 it follows that the map $\phi: X \rightarrow$ $\left(\mathbb{Z}_{P}\right)_{\infty} X$ is a $P$-equivalence, and from (i) and (ii) that $\left(\mathbb{Z}_{P}\right)_{\infty} X$ is nilpotent and $P$-local. Hence $\phi \simeq l$ and $X_{P}$ is nilpotent. Finally, $\phi \simeq \eta$ because $X$ is $\mathbb{Z}_{P \text {-good. }}$

For a space $X$ and a perfect normal subgroup $N$ of $\pi_{1}(X)$ (i.e. $H_{1}(N ; \mathbb{Z})$ $=0$ ), Quillen's plus-construction yields a homology equivalence with arbitrary twisted coefficients $q: X \rightarrow X^{+}$such that the kernel of $q_{*}: \pi_{1}(X) \rightarrow \pi_{1}\left(X^{+}\right)$ is $N$, cf. [20].

Proposition 8.5. Let $P$ be a set of primes and let $X$ be a space whose fundamental group has a perfect normal subgroup $N$ belonging to the kernel of the $P$-localizing homomorphism $\pi_{1}(X) \rightarrow \pi_{1}(X)_{P}$. Then the Quillen map $q: X \rightarrow$ $X^{+}$associated to $N$ induces a homotopy equivalence $X_{P} \simeq\left(X^{+}\right)_{P}$.

Proof. Under these hypotheses, $q_{*}: \pi_{1}(X) \rightarrow \pi_{1}\left(X^{+}\right)$is a $P$-equivalence of groups and hence $q: X \rightarrow X^{+}$is a $P$-equivalence of spaces.

The hypotheses of Proposition 8.5 are in particular satisfied if $P$ consists of a single prime $p$ and $\pi_{1}(X)$ is a finite perfect group. This provides an alternative way to construct the space $X_{P}$ in that case: First, add 2-cells and 3 -cells to $X$, so as to kill $\pi_{1}(X)$ preserving homology, and then $P$-localize the resulting simply-connected space $X^{+}$.

We conclude by explaining the effect of the functor ()$_{P}$ on a wedge of circles. Thus let $X=\bigvee_{i \in I} S^{1}$, where the index set $I$ contains at least two elements. 
Then $\left(\mathbb{Z}_{P}\right)_{\infty} X$ is an aspherical space with uncountable fundamental group [7, IV, 5.3], while $E_{P} X$ is not known in general. However, it is known that the homomorphism

$$
\gamma_{*}: \pi_{1}\left(E_{P} X\right) \rightarrow \pi_{1}\left(\left(\mathbb{Z}_{P}\right)_{\infty} X\right)
$$

is strictly surjective [5]. We next prove that $X_{P}$ is aspherical. In this context, note that $P$-localization sends countable groups to countable groups. Therefore, $\pi_{1}\left(X_{P}\right)$ is also countable, if $I$ is countable.

We first state an obvious necessary and sufficient condition for $K(G, 1)_{P}$ to be aspherical; cf. Theorem 3.2 and Lemma 5.3.

Proposition 8.6. Given a group $G$ and a set of primes $P$, the space $K(G, 1)_{P}$ is a $K\left(G_{P}, 1\right)$ if and only if the P-localizing homomorphism $l: G \rightarrow G_{P}$ induces isomorphisms

$$
l_{*}: H_{k}(G ; A) \cong H_{k}\left(G_{P} ; A\right)
$$

for all $k$ and every $P[G]-$ module $A$.

Known examples of groups $G$ satisfying the condition stated include:

(i) Nilpotent groups (Theorem 4.3).

(ii) Finite $P$-nilpotent groups [9].

(iii) The fundamental group of the Klein bottle.

(iv) All free groups (Theorem 8.7 below).

Theorem 8.7. If $F$ is a free group and $P$ a set of primes, then the P-localizing homomorphism $l: F \rightarrow F_{P}$ induces isomorphisms

$$
l_{*}: H_{k}(F ; A) \cong H_{k}\left(F_{P} ; A\right)
$$

for all $k$ and every $P[F]$-module $A$.

Proof. We use the construction of $F_{P}$ given by Baumslag [3]. Thus

$$
F_{P}=\lim _{\rightarrow} \Phi(i)
$$

for a certain direct system $\Phi$ in the category of groups, indexed by a (large) ordinal $\alpha$, with $\Phi(0)=F$, and in which each map $s_{i}: \Phi(i) \rightarrow \Phi(i+1)$ is injective. For a given index $i$, either $\Phi(i+1)=\Phi(i)$ and $s_{i}$ is the identity, or there exists a subgroup $\mathbb{Z} \subseteq U \subseteq \mathbb{Z}_{P}$ and a push-out diagram

$$
\begin{array}{ccc}
U & \stackrel{j}{\hookrightarrow} & \mathbb{Z}_{P} \\
\downarrow & & \downarrow \\
\Phi(i) & \underset{s_{i}}{\rightarrow} & \Phi(i+1)
\end{array}
$$

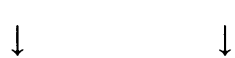

where $j$ is the inclusion and all arrows are monomorphisms. Now assume given an abelian group $A$ provided with a $P$-local action of $F_{P}$. Since homology commutes with direct limits, it suffices to show that every $s_{i}$ is an $H_{*}(\quad ; A)$-equivalence. But the inclusion $j P$-localizes and hence it is an $H_{*}(\quad ; A)$-equivalence, by Theorem 4.3. It follows, by the Mayer-Vietoris sequence associated with (8.4), that $s_{i}$ is also an $H_{*}(; A)$-equivalence, as desired. 


\section{ACKNOWLEDGMENTS}

We are indebted to the CRM of Barcelona and to the Mathematics Department of the University of Alberta for their hospitality and support. Some of the results contained in this article are extracted from the Ph.D. thesis of the firstnamed author, which was supervised by Manuel Castellet. We have benefited from discussions with Emmanuel Dror Farjoun, Peter Hilton, Guido Mislin and Markus Pfenniger.

\section{REFERENCES}

1. J. F. Adams, Localisation and completion, Lecture Notes, University of Chicago, 1975.

2. M. F. Atiyah and I. G. Macdonald, Introduction to commutative algebra, Addison-Wesley, 1969.

3. G. Baumslag, Some aspects of groups with unique roots, Acta Math. 104 (1960), 217-303.

4. A. K. Bousfield, The localization of spaces with respect to homology, Topology 14 (1975), 133-150.

5. Homological localization towers for groups and $\pi$-modules, Mem. Amer. Math. Soc. 10 (1977), No. 186.

6. __ On the p-adic completion of nonnilpotent spaces, Trans. Amer. Math. Soc. 331 (1992), 335-359.

7. A. K. Bousfield and D. M. Kan, Homotopy limits, completions and localizations, Lecture Notes in Math., vol. 304, Springer-Verlag, 1972.

8. R. Brown, Topology: A geometric account of general topology, homotopy types, and the fundamental groupoid, Halsted Press, New York, 1988.

9. C. Casacuberta, The behaviour of homology in the localization of finite groups, Canad. Math. Bull. 34 (1991), 311-320.

10. _ On the rationalization of the circle, Proc. Amer. Math. Soc. (to appear).

11. C. Casacuberta, G. Peschke and M. Pfenniger, Sur la localisation dans les catégories avec une application à la théorie de l'homotopie, C. R. Acad. Sci. Paris Sér. I 310 (1990), 207-210.

12. __ On orthogonal pairs in categories and localisation, Adams Memorial Symposium on Algebraic Topology, vol. 1, London Math. Soc. Lecture Note Ser., vol. 175, Cambridge Univ. Press, 1992, pp. 211-223.

13. P. M. Cohn, Free rings and their relations, Academic Press, 1985.

14. A. García Rodicio, Métodos homológicos en grupos P-locales, Ph.D. thesis, Universidad de Santiago de Compostela, Spain, 1986.

15. S. Gitler, Cohomology operations with local coefficients, Amer. J. Math. 85 (1963), 156-188.

16. P. Hilton, G. Mislin and J. Roitberg, Localization of nilpotent groups and spaces, NorthHolland Math. Studies, vol. 15, North-Holland, 1975.

17. P. Hilton and J. Roitberg, On the Zeeman comparison theorem for the homology of quasinilpotent fibrations, Quart. J. Math. Oxford Ser. (2) 27 (1976), 433-444.

18. G. Peschke, H-semidirect products, Canad. Math. Bull. 30 (1987), 402-411.

19. __ Localizing groups with action, Publ. Mat. 33 (1989), 227-234.

20. D. Quillen, Cohomology of groups, Actes Congrès Internat. Math., Tome 2, 1970, pp. 47-51.

21. A. Reynol, P-localization of some classes of groups, Ph.D. thesis, IME, Universidade de São Paulo, Brazil, 1987.

22. P. Ribenboim, Torsion et localisation de groupes arbitraires, Lecture Notes in Math., vol. 740, Springer-Verlag, 1978, pp. 444-456.

23. __ Equations in groups, with special emphasis on localization and torsion. I, Atti. Accad. Naz. Lincei Mem. Cl. Sci. Fis. Mat. Natur. Sez. Ia (8) 19 (1987), 23-60.

24. __ Equations in groups, with special emphasis on localization and torsion, II, Portugal. Math. 44 (1987), 417-445. 
25. S. Sehgal, Topics in group rings, Marcel Dekker, 1978.

26. B. Stenström, Rings of quotients, Grundlehren Math. Wiss. 217 (1975).

27. R. B. Warfield, Nilpotent groups, Lecture Notes in Math., vol. 513, Springer-Verlag, 1976.

Centre de Recerca Matemàtica, Apartat 50, E-08193 Bellaterra, Barcelona, Spain E-mail address: icrm1@cc.uab.es

Department of Mathematics, University of Alberta, Edmonton, Alberta, Canada T6G 2G1

E-mail address: gepe@mts.ucs.ualberta.ca 